\title{
FURTHER RESULTS ON RECURSIVE EVALUATION OF COMPOUND DISTRIBUTIONS*
}

\author{
BJøRN SUNDT and WILLIAM S. JEWELL
}

\begin{abstract}
A recent result by Panjer provides a recursive algorithm for the compound distribution of aggregate claims when the counting law belongs to a special recursive family. In the present paper we first give a characterization of this recursive family, then describe some generalizations of Panjer's result.
\end{abstract}

\section{INTRODUCTION}

Let $\mu$ be the Lebesgue or the counting measure on $(0, \infty)$, and let $\mathbf{x}_{1}, \mathbf{x}_{2}, \ldots$ be independent, identically distributed random variables (the independent severities) with cumulative distribution $F$ and generalized density $f$ :

$$
F(x)=\int_{[0, x]} f(y) d \mu(y)
$$

Let $\mathbf{n}$ be a random variable (the claim number), independent of the $\mathbf{x}_{i}^{\prime}$, defined on the non-negative integers with probabilities:

$$
p_{n}=\operatorname{Pr}(\mathrm{n}=n) \text {. }
$$

Then the generalized density $g$ of the random sum (the aggregate claims)

$$
\mathbf{s}=\sum_{i=1}^{n} \mathbf{x}_{i}
$$

(we tacitly assume $\mathbf{s}$ is zero if $\mathbf{n}$ is)

has an atom

$$
g(0)=p_{0}
$$

at zero, and for $s>0$ the form

$$
g(s)=\sum_{n-1}^{\infty} p_{n} f^{n *}(s)
$$

where $f^{n *}$ denotes the $n$-th convolution of $f$. This formula is extremely difficult to compute because of the high-order convolutions; only a few closed-form solutions are known.

* This research was supported by the Norwegian Research Council for Science and the Humanities, the Association of Norwegian Insurance Companies, and the Forschungsinstitut für Mathematik, ETH Zürich. 
PANJER (1981) has shown that, if there exist constants $a$ and $b$ such that

$$
p_{n}=p_{n-1}\left(a+\frac{b}{n}\right), \quad(n=1,2, \ldots)
$$

then

$$
g(s)=p_{1} f(s)+\int_{(0,0)}\left(a+b \frac{x}{s}\right) f(x) g(s-x) d \mu(x) .
$$

The importance of this result is that, when $f$ is discrete, the successive values of $g$ can be recursively calculated. We now consider various aspects of the relation between the recursions $(1.3)$ and (1.4), and then provide a variety of generalizations.

\section{CHARACTERIZATION OF THE COUNTING DISTRIBUTION}

The following theorem characterizes the class of counting densities $p_{n}$ satisfying (1.3); it is essentially given in JoHnson \& Korz (1969).

\section{Theorem 1}

Assume that (1.3) holds. Then ree must have one of the four cases:

$$
\begin{aligned}
& p_{n}= \begin{cases}0 & (n=0) \\
1 & (n>0)\end{cases} \\
& p_{n}=\frac{\lambda^{n}}{n !} e^{-\lambda} \quad(\lambda>0) \\
& p_{n}=\left(\begin{array}{c}
\alpha+n-1 \\
n
\end{array}\right) p^{n}(1-p)^{\alpha} \quad(\alpha>0,0<p<1) \\
& p_{n}=\left(\begin{array}{l}
N \\
n
\end{array}\right) p^{n}(1-p)^{N-n} \quad(0<p<1, N=1,2, \ldots)
\end{aligned}
$$

\section{Proof}

To avoid negative probabilities we must have $a+b \geqslant 0$. For $a+b=0$ we get the degeneratc case (2.1). For the rest of the proof we assume $a+b>0$. If $a=0$, we get the Poisson density (2.2) with $\lambda=b$. For $a>0$ we introduce $\alpha=(a+b) / a$ and get from $(1.3)$

$$
p_{n}=p_{0}(\stackrel{\alpha+n-1}{n}) a^{n}
$$

In order that $\sum_{n-1}^{\infty} p_{n}<1$, we must have $a<1$. Then we get the negative binomial (Pascal) density (2.3) with $p=a$. 
Finally, assume $a<0$. Then, to avoid negative probabilities, there must exist a positive integer $N$ such that $a+b /(N+1)=0$, that is, $N=-(a+b) / a$. With $p=-a /(1-a)$ we get the binomial density (2.4).

We have now proved the theorem.

Q.E.D.

The allowed regions for $(a, b)$ are illustrated in figure 1 , which is inspired by JoHNSON \& KOTZ (1969, p. 42).

\section{L'emark}

For the case $a<0$ Johnson \& Kotz (1969, p. 41) also develop a distribution for the case when $-(a+b) / a$ is not an integcr, by letting $p_{n}=0$ when $a+$ $(b / n)<0$. However, that distribution does not satisfy (1.4) as we then must have that (1.3) holds for all $n>0$. A modified version of (1.4) allowing such "generalized binomial" distributions will be given in Section 5. However, this version seems in most cases to be more complicated than direct computation of (1.2). For the binomial distribution we have that $\operatorname{Pr}(\mathbf{n}>N)=0$, but as $\left(\begin{array}{l}N \\ n\end{array}\right)=0$ for $n>N$ we can let $p_{n}$ be defined by $(2.4)$ for all the non-negative integers.

\section{GENERALIZATIONS}

We first introduce some notation: if $z_{1}, z_{2}, \ldots$ are given quantities, then we let

$$
z_{n \Sigma}=\sum_{i-1}^{n} z_{i}
$$

denote the sum of the first $n$ elements.

Assume there exists a function $h:\{(x, s): 0<x<s\} \rightarrow \mathbb{R}$, satisfying the condition that

$$
\begin{aligned}
& \mathbb{E}\left(h\left(\mathbf{x}_{1}, s\right) \mid \mathbf{x}_{n \Sigma}=s\right)=m_{n} \\
& \text { are independent of } s .
\end{aligned} \quad(n=2,3, \ldots)
$$

Then we have the following generalization of Panjer's result:

\section{Theorem 2}

If

$$
p_{n}=p_{n-1} m_{n}, \quad(n=2,3, \ldots)
$$

with the sequence $\left\{m_{n}\right\}$ satisfying (3.1), then

$$
g(s)=p_{1} f(s)+\int_{(0,0)} h(x, s) f(x) g(s-x) d \mu(x) \cdot(s>0)
$$




\section{Proof}

We have for $s>0$

$$
\begin{aligned}
g(s)= & \sum_{n-1}^{\infty} p_{n} f^{n *}(s)= \\
& p_{1} f(s)+\sum_{n-2}^{\infty} p_{n-1} m_{n} f^{n *}(s)= \\
& p_{1} f(s)+\sum_{n-2}^{\infty} p_{n-1} \int_{(0,0)} h(x, s) f(x) f^{(n-1) *}(s-x) d_{\mu}(x)= \\
& p_{1} f(s)+\int_{(0,0)} h(x, s) f(x)\left[\sum_{n=1}^{\infty} p_{n} f^{n *}(s-x)\right] d_{\mu}(x)= \\
& p_{1} f(s)+\int_{(0,0)} h(x, s) f(x) g(s-x) d \mu(x) .
\end{aligned}
$$

Q.E.D.

It is clear that if the functions $h_{1}$ and $h_{2}$ both satisfy (3.1), then for all constants $c_{1}$ and $c_{2}$ the function $c_{1} h_{1}+c_{2} h_{2}$ satisfies (3.1).

For all constants $a$ and $b$ we clearly have

$$
F\left(a+b \frac{\mathbf{x}_{1}}{s} \mid \mathbf{x}_{n \Sigma}=s\right)=a+\frac{b}{n}, \quad(n=2,3, \ldots)
$$

independent of $s$. Hence the kernel in (1.3),

$$
h(x, s)=a+b \frac{x}{s},
$$

is a special case of (3.1) with

$$
m_{n}=a+\frac{b}{n} . \quad(n=2,3, \ldots)
$$

The following example gives a distribution satisfying (3.1) with $m_{n}$ satisfying (3.5), but not covered by Panjer's result.

\section{Example 1}

Consider the logarithmic counting density

$$
p_{n}=0 \quad(n=0)
$$

$$
(0<p<1)
$$

$$
\frac{1}{|\ln (1-p)|} \frac{p^{n}}{n} \quad(n=1,2, \ldots)
$$


Then we have

$$
p_{n}=p_{n-1}\left(1-\frac{1}{n}\right), \quad(n=2,3, \ldots)
$$

that is, $m_{n}=1-(1 / n) ; a=1 ; b=-1$, and for $s>0$ we obtain

$$
g(s)=p_{1} f(s)+\int_{(0,0)}\left(1-\frac{x}{s}\right) f(x) g(s-x) d \mu(x) .
$$

The difference from Panjer's result is that (1.3) does not hold for $n=1$.

Theorem 3

Assume that (3.1) is satisfied for the distributions given by

$$
\operatorname{Pr}\left(\mathbf{x}_{i}=1\right)=1-\operatorname{Pr}\left(\mathbf{x}_{i}=2\right)=p . \quad(0 \leqslant p \leqslant 1)
$$

Then there must exist constants $a$ and $b$ such that (3.5) is satisfied.

Proof

For $p=1$ and $p=0$ we get

$$
\begin{aligned}
& m_{n}=h(1, n) \\
& m_{n}=h(2,2 n)
\end{aligned}
$$

respectively.

Assume $p \varepsilon(0,1) ; u=2,3, \ldots ; n=u, u+1, \ldots, 2 u$. Then

$$
\frac{f(y) f^{(n-1) *}(2 u-y)}{f^{n *(2 u)}}=\frac{\left(\begin{array}{l}
n-1 \\
2 u-y-n+1
\end{array}\right)}{\left(\begin{array}{l}
n \\
2 u-n
\end{array}\right)} . \quad(y=1,2)
$$

By using (3.7), (3.8), and (3.9) in

$$
\begin{aligned}
m_{n} & =\frac{f(1) f^{(n-1) *}(2 u-1)}{f^{n *}(2 u)} h(1,2 u) \\
& +\frac{f(2) f^{(n-1) *}(2 u-2)}{f^{n *}(2 u)} h(2,2 u)
\end{aligned}
$$

we obtain

$$
m_{n}=a_{u}+\frac{b_{u}}{n}
$$

with

$$
a_{u}=2 m_{2 u}-m_{u}, \quad b_{u}=2 u\left(m_{u}-m_{2 u}\right) .
$$


As

$$
\begin{aligned}
& m_{u+1}=a_{u+1}+\frac{b_{u+1}}{u+1}=a_{u}+\frac{b_{u}}{u+1}, \\
& m_{u+2}=a_{u+1}+\frac{b_{u+1}}{u+2}=a_{u}+\frac{b_{u}}{u+2},
\end{aligned}
$$

we must have $a_{u+1}=a_{u}$ and $b_{u+1}=b_{u}$ for all $u$, that is, there exist constants $a$ and $b$ such that (3.5) is satisfied.

Q.E.D.

Theorem 3 says that if $(3.1)$ is to hold for a class of two-point distributions $F$, the sequence $\left\{m_{n}\right\}$ must satisfy $(3.5)$. This result clearly implies that if (3.1) is to hold for all distributions on $(0, \infty)$, the sequence $\left\{m_{u}\right\}$ must satisfy (3.5). Because of this fact we restate Theorem 2 for this particular class of counting distributions.

\section{Theorem 2}

If

$$
p_{n}=p_{n-1}\left(a+\frac{b}{n}\right), \quad(n=2,3, \ldots)
$$

then for all severity distributions $F$ we have

$$
g(s)=p_{1} f(s)+\int_{\langle 0, \text {, })}\left(a+b \frac{x}{s}\right) f(x) g(s-x) d \mu(x) . \quad(s>0)
$$

We close this section by comparing the class of counting distributions defined by (1.3) (that is, the class given in Theorem 1) to the class defined by (3.10). Clearly the latter class contains the former one. As in the latter class the recursion may start at one, the restriction $a+b \geqslant 0$ may for $a>0$ be replaced by the weaker condition $a+b / 2 \geqslant 0$. Hence, the permitted parameter space is now increased by the dotted region of figure 1 .

As $p_{0}$ may now be chosen (relatively) freely, the counting distribution is no longer uniquely determined by $(a, b)$. For $(a, b)$ being in the permitted region for recursion (1.3), excluding the line $a+b=0$, the permitted class consists of the distributions given by

$$
p_{n}= \begin{cases}\rho+(1-p) \pi_{0} & (n=0) \\ (1-p) \pi_{n}, & (n=1,2, \ldots)\end{cases}
$$

where $\left\{\pi_{n}\right\}$ is a counting distribution satisfying (1.3), and $p$ is chosen such that $p \leqslant 1$ and $p_{0} \geqslant 0 . p_{n}$ clearly satisfies (3.10) with the same $(a, b)$ as for $\pi_{n}$. 


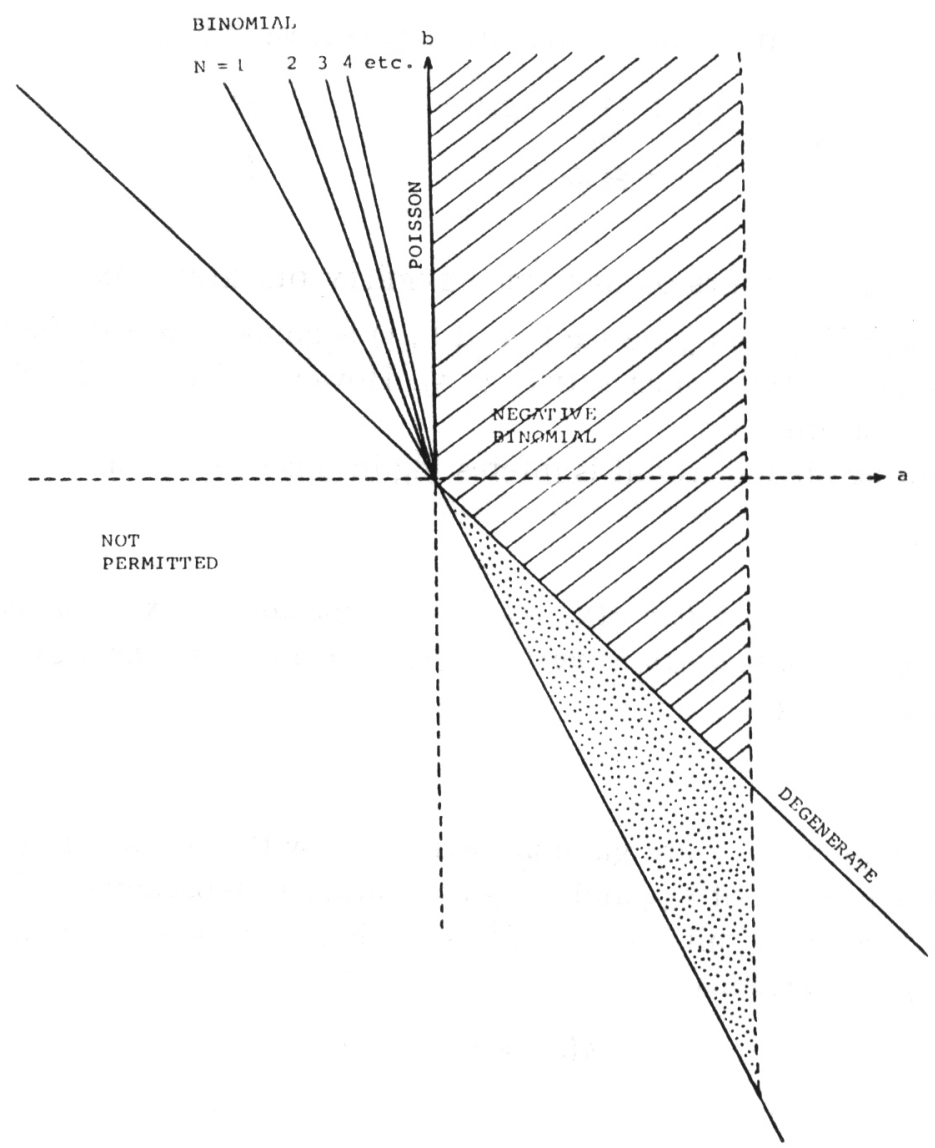

Fig 1 permitted $(a, b)$ parameter space for recurbion ( 13 ) (The dotted ared clenotes the increase obtained by recursion $(310))$

In the discrete case ( 311 ) may under the piesent conditions be witten as

$$
g(s)=(a+b) \rho F(s)+\sum_{x=1}^{\dot{c}}\left(a+b \frac{x}{s}\right) f(x) g(s-x) \quad(s>0)
$$

For $a+b=0$ the peimitted class of counting distributions is given by (3 12), with the obvious iestrictions on $p$, and $p_{n}$ given by (36)

A counting distribution $\left\{p_{n}\right\}$ of the form (3 12) may be inter pieted as a weighted (in a general sense, as $p$ may be negative) distribution of the distribution $\left\{\pi_{n}\right\}$ and a distribution concentiated at zero Then the aggregate clains distribution must be the analogous weighted distribution of aggregate clarms distributions, and if the aggiegate claims distribution $g_{\pi}$ con esponding to $\pi_{n}$ is known, we 
may find the aggregate claims distribution $g_{p}$ corresponding to $p_{n}$ by

$$
g_{p}(s)= \begin{cases}p+(1-p) g_{\pi}(0) & (s=0) \\ (1-p) g_{\pi}(s) . & (s>0)\end{cases}
$$

\section{RESULTS ON SPECIFIC SEVERITY DISTRIBUTIONS}

From (3.4) and Theorem 3 we see that if (3.1) is going to be satisfied for all $F$, then the sequence $\left\{m_{n}\right\}$ must satisfy (3.5). However, for specific classes of $F$ there may exist other $m_{n}$.

The following obvious result is interesting in this connection.

\section{Theorem 4}

Let $v$ be a function such that $v\left(\mathbf{x}_{1}, \mathbf{x}_{n \Sigma}\right)$ is independent of $\mathbf{x}_{n \Sigma}$ for all $n$. Then (3.1) holds for any h that can be written $h(x, s)=k(v(x, s))$ with $\mathbb{E}\left(h\left(\mathbf{x}_{1}, \mathbf{x}_{n \Sigma}\right)\right)$ existing for $n=2,3, \ldots$

\section{Example 2}

Assume that $\mathbf{x}_{1}, \mathbf{x}_{2}, \ldots$ are gamma-distributed with parameters $(\alpha, v)$. Then $\mathbf{x}_{1} / \mathbf{x}_{n \Sigma}$ is independent of $\mathbf{x}_{n \Sigma}$ and beta-distributed with parameters $(v,(n-1) v)$. Hence, by Theorem 4 , all $h(x, s)=k(x / s)$ with $ङ\left(k\left(\mathbf{x}_{1} / \mathbf{x}_{n \Sigma}\right)\right)$ existing for all $n$ satisfy (3.1). In particular, if

$$
k(z)=z^{u}(1-z)^{v}
$$

we get

$$
m_{n}=\frac{\Gamma(n v) \Gamma^{\prime}(v+u) \Gamma((n-1) v+v)}{\Gamma((n-1) v) \Gamma(v) \Gamma(n v+u+v)}
$$

For $v=o$ and $u$ positive integer this gives

$$
m_{n}=\prod_{i=0}^{n-1} \frac{\nu+i}{n v+i}=\sum_{i=0}^{n-3} \frac{a_{i}}{n v+i}
$$

for some $a_{0}, \ldots, a_{u-1}$ independent of $n$. Hence, for any positive integer $u$ there exist constants $c_{1}, \ldots, c_{u+1}$ such that

$$
k(z)=\sum_{i=2}^{N+1} c_{i} z^{i}
$$

gives

$$
m_{n}=\frac{1}{n v+u}
$$




\section{Example 3}

Assume that the counting density is hypergeometric

$$
p_{n}=\frac{\left(\begin{array}{c}
m \\
n
\end{array}\right)\left(\begin{array}{c}
M-m \\
N-n
\end{array}\right)}{\left(\begin{array}{c}
M \\
N
\end{array}\right)}
$$

where the positive integer parameters $(m, M, N)$ satisfy $N<M ; m \leqslant M-N$. For $n>0$ we have

$$
p_{n}=p_{n-1} \frac{(n-n+1)(N-n+1)}{n(M-n-N+n)}
$$

which maly be written

$$
p_{n}=p_{n-1}\left(a+\frac{b}{n}+\frac{c}{n+M-m-N}\right)
$$

with

$$
\begin{aligned}
& a=1, \\
& b=-\frac{(m+1) \frac{(N+1)}{N-M+m},}{c=-\frac{(M-m+1)(N-M-1)}{N-M+m} .} .
\end{aligned}
$$

Now, assume that the $\mathbf{x}_{i}^{\prime}$ s are gamma-clistributed with parameters $(\alpha, v)$, where $y$ is a positive integer. As we may write

$$
\overline{n+M-m-N}=\frac{c \nu}{m \nu+(M-m-N) \nu},
$$

by Example 2 we can find a function $h$ such that Theorem 2 is satisficd.

The extension to the eccentric hypergeometric distribution (see SVERDruP (1976), with counting density

$$
p_{n}^{\prime}=\frac{p_{n} \lambda^{n},}{\sum_{n^{\prime}} p_{n^{\prime}} \lambda^{n^{\prime}}}, \quad(\lambda>0)
$$

where $p_{n}$ is given by (4.1), is obvious.

Similar approaches are possible for the following counting distributions, described in Jomnson \& Kotz (1969): the displaced Poisson distribution (p. 113); and the Yule distribution with generalizations (pp. 244-251). 


\section{RECUIRSION ON A LIMITED RANGE}

In the previous cases we have assumed that the $p_{n}$ can be computed recursivcly for $n>1$. The following Theorem 5 extends this to the case when the recursion holds only for $n>K$ with $K \geqslant 1$.

Let

$$
g_{K}(s)=\sum_{n-K}^{\infty} p_{n} f^{n *}(s)
$$

Then

$$
g(s)=\sum_{n-0}^{K-1} p_{n} f^{n *}(s)+g_{K}(s)
$$

Theorem 5

Assume that

$$
p_{n}=p_{n-1} m_{n} . \quad(n=K+1, K+2, \ldots)
$$

with $m_{n}$ given as in (3.1). Then

$$
g_{K}(s)=p_{K} f^{K *}(s)+\int_{(0,1)} h(x, s) f(x) g_{K}(s-x) d \mu(x) .
$$

(The proof goes as in Theorem 2 and is omitted.)

The difference from the underlying assumptions of Theorem 2 is that (3.1) and (3.2) do not need to hold for $n \leqslant K$. If (3.1) holds for all $n \geqslant 2$, insertion of

$$
g_{K}(s)=g(s)-\sum_{n-1}^{k-1} p_{n} f^{n *}(s) \quad(s>0)
$$

in $(5.1)$ gives the final recursion:

$$
\begin{aligned}
g(s) & =p_{1} f(s)+\sum_{n-2}^{k}\left(p_{n}-p_{n-1} m_{n}\right) f^{n *(s)} \\
& +\int_{(0,0)} h(x, s) f(x) g(s-x) d \mu(x) . \quad(s>0)
\end{aligned}
$$

(The summation is zero if $K=1$.) Compared to (3.3) we have now got the summation as a correction term, since this would be zero if $p_{n}-p_{n-1} m_{n}=0$ for $\mathrm{n}=2, \ldots, K$.

For the special case of Theorem 5 with $p_{0}=p_{1}=\ldots=p_{K-1}=0$ (truncation from below) $g_{K}(s)=g(s)$, and (5.1) gives

$$
g(s)=p_{K} f^{K *}(s)+\int_{(0,0)} h(x, s) f(x) g(s-x) d \mu(x) .
$$


We shall now see what happens if the counting distribution is truncated from above. Assume

$$
\begin{aligned}
p_{n} & =0 & & (n=0, \ldots, K-1) \\
& =p_{n-1} m_{n} & & (n=K+1, \ldots, L) \\
& =0 & & (n=L+1, \ldots) .
\end{aligned}
$$

Then for $s>0$ we get

$$
\begin{aligned}
g(s) & =p_{K} f^{K *}(s)-p_{L} m_{L+1} f^{(L+1) *}(s) \\
& +\int_{(0, \Omega)} h(x, s) f(x) g(s-x) d \mu(x) .
\end{aligned}
$$

Unfortunately, in this formula we need high-order convolutions of $f$. These can be rather complicated to compute, except for some cases where we have simple closed-form expressions (gamma, Poisson, binomial, negative binomial distributions). In some cases the factor $p_{L} m_{L+1}$ makes the correction term negligible. Another possibility is for large $L$ to approximate $f^{(L+1) *}$ by a (possibly discretized) normal density. Otherwise it is probably more efficient to compute $g$ from the basic definition (1.1).

\section{EXTENSION TO NON-POSITIVE DISCRETE VALUES}

We now leave the assumption that the $\mathbf{x}_{i}^{\prime}$ s are distributed on $(0, \infty)$ and assume that they are distributed on the set of all integers:

$$
f(x)=\operatorname{Pr}(\mathrm{x}=x) . \quad(x=\ldots-2,-1,0,1,2, \ldots)
$$

Then (1.1) must be replaced by

$$
g(0)=p_{0}+\sum_{n=2}^{\infty} p_{n} f^{n *}(0)
$$

We further assume that the counting distribution satisfies the recursion (1.3), and analogously to Theorem 2 we obtain

$$
s g(s)=\sum_{x=-\infty}^{+\infty}(a s+b x) f(x) g(s-x) .
$$

If $\mathbf{x}_{j}$ only takes on zero plus positive values, so does $\mathbf{s}$; then $f^{n *}(0)=[f(0)]^{n}$, and the sum in (6.1) can be carried out explicitly (see the probability generating function for the counting distribution in JOHNSON \& KOTz (1969)). We then get the recursive system 
(6.3)

$$
\begin{aligned}
g(0) & =\left[\frac{1-a f(0)}{1-a}\right]^{-\left(\frac{a+b}{a}\right)} & & (a \neq 0) \\
& =c^{-b[1-f(0)] ;} & & (a=0)
\end{aligned}
$$

$$
g(s)=\left(\frac{1}{1-a f(0)}\right) \sum_{x=1}^{\infty}\left(a+b \frac{x}{s}\right) f(x) g(s-x) .
$$

The case where the $x_{i}$ can take on negative values is difficult because one cannot, in general, find suitable starting values for $s$ in (6.2).

However, in the case where $p_{n}$ is Poisson with parameter $7 .(2.2)$, the density $g$ can be computed by two applications of $(6.3)$ plus a convolution. Let

$$
\begin{aligned}
& \mathbf{x}_{i}^{+}=\max \left(0, \mathbf{x}_{i}\right) \\
& \mathbf{x}_{i}^{-}=\max \left(0,-\mathbf{x}_{i}\right), \quad(i=1,2, \ldots)
\end{aligned}
$$

and we have

$$
\mathbf{s}^{+}=\sum_{i=1}^{n} \mathrm{x}_{i}^{+} ; \quad \mathbf{s}^{-}=\sum_{i=1}^{n} \mathrm{x}_{i}^{-}
$$

$$
\mathbf{s}=\mathbf{s}^{+}-\mathbf{s}^{-} \text {. }
$$

André Dubey has pointed out to us that when $\mathrm{n}$ is Poisson distributed, then $\mathbf{s}^{+}$and $\mathbf{s}^{-}$are independent. Let $\mathbf{x}_{i}^{+}$and $\mathbf{x}_{i}^{-}$have densitics $f^{+}$and $f^{-}$, respectively, and $\mathbf{s}^{+}$and $\mathbf{s}^{-}$have densities $g^{+}$and $g^{-}$, respectively. Then $g^{+}$and $g^{-}$are computed independently, using (6.3), with $a=0, b=\lambda$, and the corresponding $f^{+}$or $f^{-}$. Then $g$ for the total sum is computed by the convolution

$$
g(s)=\sum_{x=\max (0.0)}^{\infty} g^{+}(x) g-(x-s) .
$$

(6.2) can, in principle, also be solved for $p_{n}$ binomial, if $f(x)$ is defined over $(-K,-K+1, \ldots)$, for in that case there is a largest negative value of the sum, $s=-N K$, and (6.2) can be rcarranged into a true recursive form.

Remembering that $p=-a /(1-a)$ and $N=-(a+b) / a$, we get the recursive system:

$$
\text { (6.7) } \begin{array}{rlrl}
g(s) & =0 & & (s<-N K) \\
& =[p f(-K)]^{N} & & (s=-N K) \\
= & & (s>-N K) .
\end{array}
$$


Of course, if $K$ is very large, there are obvious problems with round-off crror accumulation, especially if $f(-K)$ and the nearby values are very small. We remind the reader that this problem can occur with any recursive scheme clescribed in this paper where the range of discrete severities is large.

There remains the case of $p_{n}$ negative binomial (2.3) for which it does not seem possible to give a simple procedure for negative $\mathbf{x}_{i}^{\prime} \mathrm{s}$. Of course, in this and in the other cases, one can think of various iterative schemes for (6.2) which would converge to the correct density.

\section{REFERENCES}

Johnson, N. L. \& Korz, S. (1969). Discrete distributions. Houghton Miflin, Boston.

PANJER, H. H. (1981). Recursive cvaluation of a family of compound distributions. Astin Bulletin 12.

Sviridrup, E. (1976). Programs on HP-25 desk calculator of mathematical functions for use in statistics. Statistical Research Report 1976-7. Institute of Mathematics. University of Oslo. 